\title{
The Many Connections Between Atom Probe and Electron Microscopy
}

\author{
Thomas F. Kelly ${ }^{1}$ \\ 1. CAMECA Instruments, Inc., 5470 Nobel Drive, Madison, WI 53711 USA.
}

Though it may not seem so at first blush, there are many connections between electron microscopy and atom probe tomography. The genesis of both can be traced to at least the early twentieth century when an experimental demonstration of the existence of atoms in crystals (and of the wave nature of x-rays) was achieved in 1912 by von Laue [1,2]. The theory of the wave nature of matter had been put forward by de Broglie in 1923 [3] and a theory of the focusing action of an electromagnetic coil was formulated by Busch [4] in 1927. At the Technische Hochschule Berlin-Charlottenburg (now Technische Univerität Berlin or Technical University of Berlin), two projects had started in the early 1930s. A graduate student named Ernst Ruska, Fig. 1a, working under Professor Max Knoll had started experiments on the lensing action of magnetostatic fields on electron beams both to test Busch's theory and to test concepts for making an electron microscope. Another student named Erwin Müller, Fig. 1b, working under Professor Gustav Hertz, was using high electrostatic fields applied to material surfaces to extract electron beams. It would be very interesting to know if either sensed that these efforts at the same institution would eventually lead to the first images of atoms: Müller using field ion microscopy in 1955 [5,6] and Crewe et al. using scanning transmission electron microscopy in 1970 [7]. As we will see later, these two techniques will likely be joined again into a single instrument.

During his career, Müller developed the Field Emission Microscope (FEM), the Field Ion Microscope (FIM), and the Atom Probe. Ruska developed the first electron microscope that surpassed the image resolution of light microscopy. It is said by some experts that Ruska and Müller were in line to share a Nobel Prize when Müller died unexpectedly in 1977. Much of Müller's early work contributed to the development of field emission electron sources which dominate the high end of electron microscopes today. There is also a connection between Müller's laboratory and the third atomic-scale imaging technique, scanning probe microscopy. Russel Young studied as a post-doctoral researcher at Penn State with Müller and learned about electron tunneling and high field science. He went on to the National Bureau of Standards to develop the predecessor of the scanning tunneling microscope, the topographiner [8].

Because of the size of atom probe specimens, TEM and SEM have always been used to image atom probe specimens. Fig. 2 [9] is an example where transmission Kikuchi diffraction patterns are recorded on an atom probe specimen during preparation and are used to locate a grain boundary for atom probe analysis. The result is a superior analysis with greater certainty which was obtained in far less time than previously. This information has been instrumental in development of atom probe theory and has been used as important feedback for atom probe image reconstructions. There is interest today in combining TEM with atom probe [10] to facilitate combination of their respective data and to realize synergies. It should be possible to eliminate distortions in atom probe reconstructions and achieve atomic-scale tomography [10] with information on the shape of the specimen apex during the atom probe experiment so that every atom may be positioned in three dimensions with atom-scale accuracy and precision. Thus, we have come full circle in the past 75 years: field emission research has made it possible to make better electron microscopes which are used to make better images of field evaporation specimens and achieve atomic-scale analytical tomography by exploiting the best of both microscopies. Ruska and Müller would be proud. 


\section{References:}

[1] W Friedrich, P Knipping, and M von Laue, Sitzungber K Bayer Akad Wiss. 1912, (1912) p. 303.

[2] W Friedrich, P Knipping, M von Laue, Radium 10, (1913) p. 47.

[3] L de Broglie, Nature 112, (1923) p. 540.

[4] H Busch, Arch Elektrotechnik. 18, (1927) p. 583.

[5] K Bahadur, Ph. D. Thesis, The Pennsylvania State University, 1955.

[6] EW Müller and K Bahadur, Phys. Rev., 102, no. 3 (1956), p. 624.

[7] AV Crewe, J Wall and J Langmore, Science, 168, no. 3937 (1970), p. 1338.

[8] RD Young, Phys. Today, 24, no. 11 (Nov. 1971) p. 42.

[9] Y Chen et al., Microsc. Microanal. 22, no. S3, (2016) p. 682.

[10] TF Kelly, Microsc. Microanal., 23 (2017) p. 34.

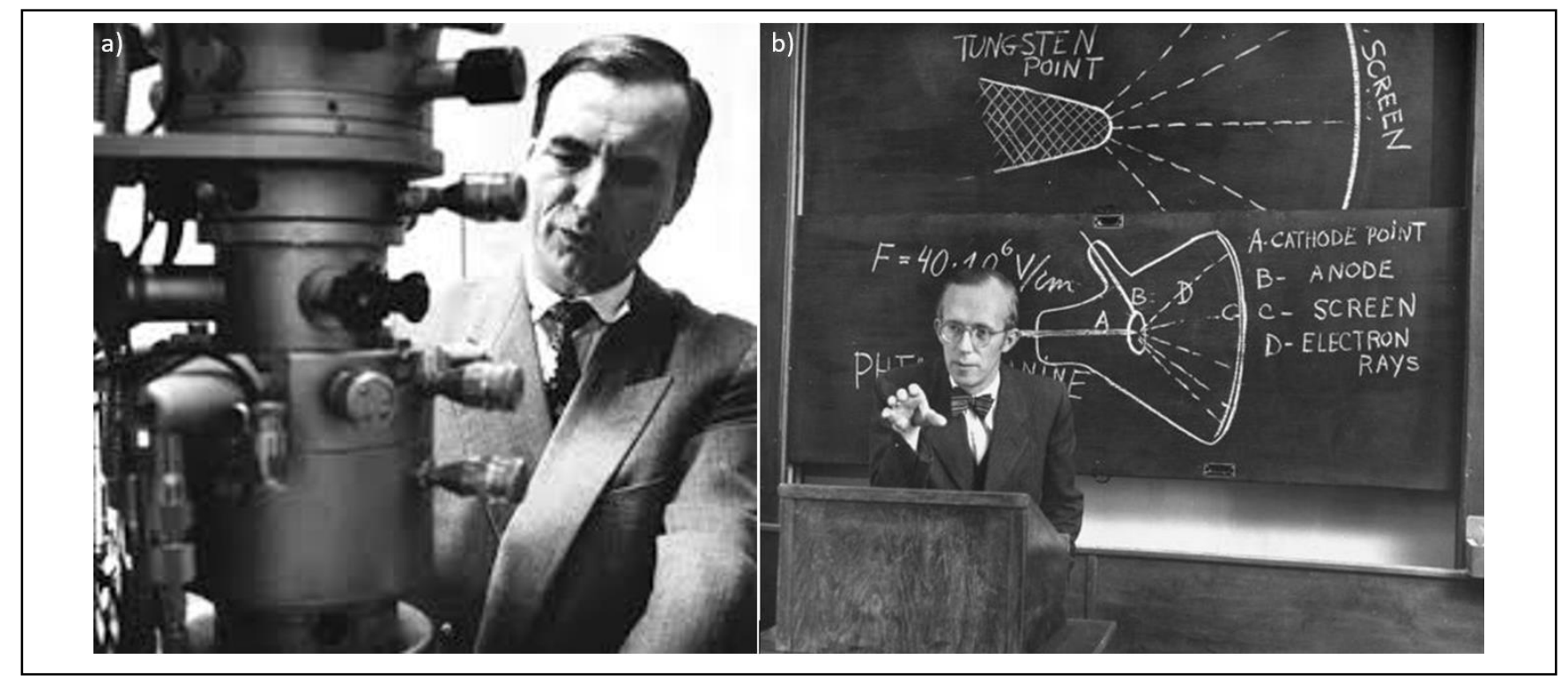

Figure 1. Ernst Ruska at the column of an electron microscope. b) Erwin W. Müller lecturing at the Pennsylvania State University.

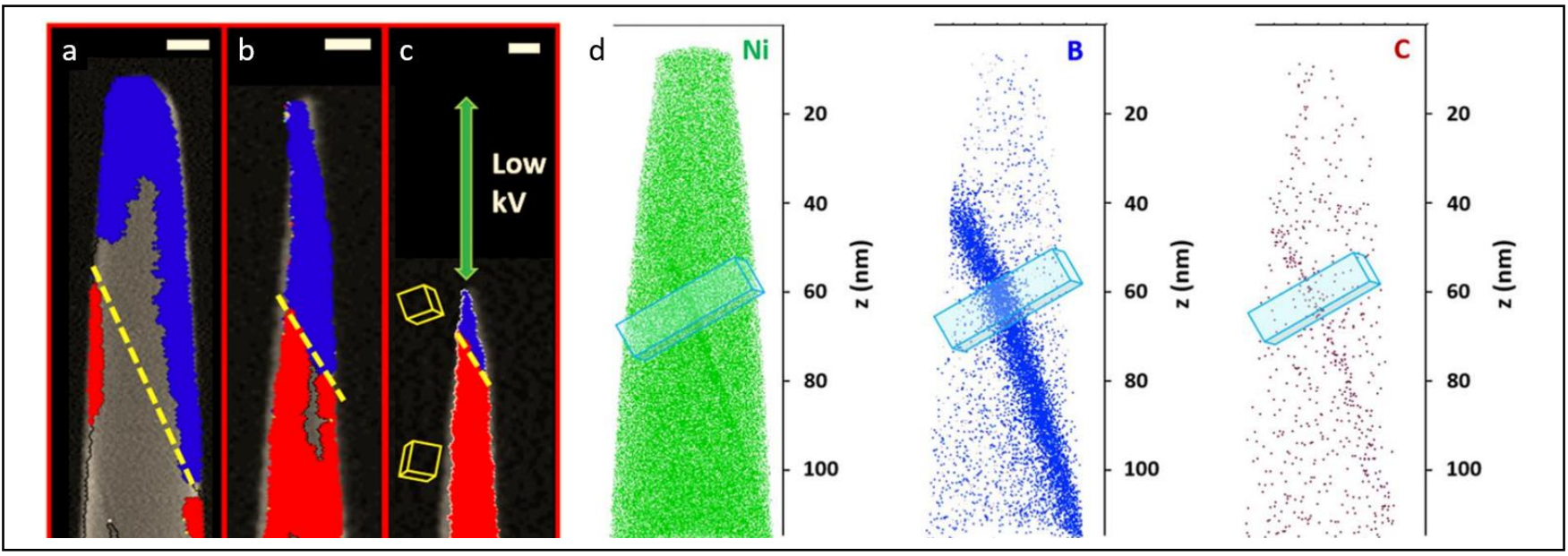

Figure 2. Analysis of Inconel 600 from [9]. a-c) Transmission Kikuchi patterns during the milling process taken at $30 \mathrm{kV}$ used in preparation of atom probe specimens of a grain boundary.. Scale bar is 200nm. c) Final milling was performed at $2 \mathrm{kV}$ to remove damage. d) atom maps of $\mathrm{Ni}, \mathrm{B}$, and $\mathrm{C}$ showing segregation at the grain boundary identified by the dashed yellow line. The misorientation of the two grains is $50^{\circ}$. 